\title{
Aberrant Cx43 Expression and Mislocalization in Metastatic Human Melanomas
}

\author{
Katanya C. Alaga ${ }^{1}$, Melissa Crawford ${ }^{2}$, Lina Dagnino ${ }^{2}$, and Dale W. Laird ${ }^{1}{ }^{2}$ \\ 1. Department of Anatomy and Cell Biology, The University of Western Ontario, London, ON, Canada; \\ 2. Department of Physiology and Pharmacology, The University of Western Ontario, London, ON, Canada. \\ $\triangle$ Corresponding author: Dale W. Laird, PhD., Department of Anatomy and Cell Biology, University of Western Ontario, London, Ontario, N6A 5C1 \\ 519-661-2111 x86827 Fax: 519 850-2562 Dale.Laird@schulich.uwo.ca \\ (c) Ivyspring International Publisher. This is an open access article distributed under the terms of the Creative Commons Attribution (CC BY-NC) license \\ (https://creativecommons.org/licenses/by-nc/4.0/). See http://ivyspring.com/terms for full terms and conditions.
}

Received: 2016.11.30; Accepted: 2017.03.06; Published: 2017.04.09

\begin{abstract}
At present, it is unclear if melanocytes contain $\mathrm{C} x 43$ gap junctions and whether $\mathrm{Cx} 43$ expression is regulated in melanoma onset and progression. To this end, we cultured pure populations of mouse melanocytes and found that they had no detectable $\mathrm{C} \times 43$ and exhibited an inability for dye transfer indicating they were devoid of functional gap junctions. Given the evidence that melanomas acquire the expression of other connexin isoforms during tumor progression, we assessed if $\mathrm{C} \times 43$ was also expressed and assembled into gap junctions at any stage of human melanoma onset and progression to distant metastases. Nearly all primary melanomas within the epidermis lacked Cx43. In contrast, nodal metastases expressed low levels of Cx43 which was markedly higher in distant metastases that had invaded vital organs. Importantly, in all stages of melanoma progression, Cx43 could be detected in intracellular compartments but was rarely assembled into gap junctions indicative of functional gap junction channels. Overall, these studies suggest that melanocytes do not form $\mathrm{Cx}_{4} 43$ homocellular gap junctions and even though $\mathrm{C} \times 43$ levels increase during melanoma progression, this connexin rarely assembles into gap junction structures.
\end{abstract}

Key words: connexin; gap junctions; gap junctional intercellular communication; Cx43; melanoma.

\section{Introduction}

Connexins encompass a large family of integral membrane proteins that form gap junction channels to allow for the intercellular exchange of metabolites and small molecules $(<1 \mathrm{kDa})$, termed gap junctional intercellular communication (GJIC) [1]. Connexin proteins oligomerize into hexameric channels (termed connexons), which translocate to the plasma membrane where they can dock with a connexon from an adjacent cell to form a gap junction channel for direct exchange of cytosolic signaling molecules [2]. The expression profile of connexins is tissue-specific and can be altered in many pathologies including skin cancers, highlighting their complex regulation during disease [3].

Despite the vast array of connexin isoforms expressed throughout the keratinocyte layers, surprisingly little is known about the connexin profile of melanocytes [4, 5]. This sparsity of connexin knowledge also extends to mouse melanocytes even though mice have been used as experimental models for in vivo melanoma progression. One study suggests that human melanocytes exhibited GJIC with co-cultured keratinocytes a process that was mediated by the expression of E-cadherin [5]. These same authors went on to show that a melanoma cell line lost the ability to communicate with keratinocytes but this was replaced with the acquired ability to express $\mathrm{N}$-cadherin and establish GJIC with fibroblasts [5]. Tissue microarray analysis of primary cutaneous human melanomas revealed increased Cx43 expression in comparison to melanocytic nevi, pointing to a possible role for $\mathrm{C} \times 43$ as a tumor facilitator [6]. This notion was supported by Sargen and colleagues who suggested that primary human 
melanomas exhibited more Cx43 immunostaining than benign nevi although they did not assess melanomas that had invaded nodes or had metastasized to distant organs [7]. In contrast, Haass and colleagues reported no Cx43 staining in human melanocytic nevi and malignant melanomas in situ questioning the extent of $\mathrm{C} \times 43$ regulation in melanoma tumorigenesis [8]. In all of these studies no clear distinctions were made between Cx43 expression and its in vivo assembly into prototypical gap junctions indicative of its functioning in cell-cell communication. Given these conflicting observations, a thorough characterization of the expression and localization of $\mathrm{C} \times 43$ throughout the different stages of human melanoma progression remains to be investigated.

\section{Materials and Methods}

\section{Primary Mouse Keratinocyte and Melanocyte Cultures}

The dorsal skin was collected from wild type mouse neonates (2-3 days of age) as previously described [9]. Isolation of primary mouse melanocytes was accomplished as previously described [10], with the following modifications. Following dispase incubation, 6-8 epidermises were separated, minced, and transferred to tubes containing $0.25 \%$ Trypsin/EDTA solution. The epidermis was then incubated on a rocker at $37^{\circ} \mathrm{C}$, and then the Trypsin/EDTA was neutralized. The suspension was passed through a $70-\mu \mathrm{m}$ strainer, and centrifuged for 10 mins at $1000 \mathrm{rpm}$. The pellet was re-suspended in 5 $\mathrm{ml}$ of Clonetics Melanocyte Growth Medium-4 (MGM-4 Bullet Kit, CC-3249). Cells $\left(1 \times 10^{6}\right)$ were plated on $60 \mathrm{~mm}$ dishes. Melanocytes were passaged when they were $80 \%$ confluent. Cells analyzed by immunohistochemistry were plated on coverslips coated with rat-tail collagen type 1 (BD Biosciences, 354236). Coverslips with attached melanocytes were removed, washed in PBS ( $\mathrm{pH}$ 6.8) and fixed in $4 \%$ PFA for 20 minutes at $25^{\circ} \mathrm{C}$. PFA was removed and fixed cells were washed three times in PBS ( $\mathrm{pH}$ 6.8), prior to incubation with $0.1 \%$ L-3,4dihydroxyphenylalanine (L - DOPA) (Alfa Aesar, A11311) for $4 \mathrm{hrs}$ at $37^{\circ} \mathrm{C}$, as previously described [11]. Coverslips were subsequently washed with PBS ( $\mathrm{pH}$ 6.8) before being mounted on glass slides with Permount and five images were taken for each passage using a LSM 800 Zeiss confocal microscope using a 40x lens. Images were blinded to the investigator and melanocyte purity was calculated by measuring the percent yield of L-DOPA positive cells (dark pigmented cells) verses total cells in an image area. For primary keratinocyte cultures, keratinocytes were cultured as previously described by Churko et al. 2012 [9]. To induce keratinocyte differentiation, primary cells were placed in $1.4 \mathrm{mM}$ calcium $24 \mathrm{hrs}$ after isolation, and maintained for an additional 24 hrs before use [9].

In some cases, keratinocytes and/or melanocytes were washed in PBS, and fixed in 4\% PFA for 20 mins at $25^{\circ} \mathrm{C}$. Cells were labeled with either mouse anti-tyrosinase related protein 1 (TRP1, 1:200; Abcam 3312), mouse anti-E-cadherin (1:200; BD Transduction Labs, 610182) or rabbit anti-Cx43 (Sigma Cat\# C6219, $1: 400)$ overnight at $4^{\circ} \mathrm{C}$. Subsequently, cells were incubated with Alexa Fluor 555-conjugated anti-rabbit (1:400; Molecular Probes) or Alexa Fluor 488-conjugated anti-mouse (1:400; Molecular Probes) for 1 hour at $25^{\circ} \mathrm{C}$ followed by Hoescht 33342 staining for 10 mins at $25^{\circ} \mathrm{C}$ (1:1000; Molecular Probes, H3570) prior to mounting using Airvol and imaged by confocal microscopy [12].

\section{Calcein-AM Dye Transfer}

P2 primary mouse melanocytes and rat epidermal keratinocytes (REKs), which were previously isolated and characterized [13], were grown to confluence on glass bottom dishes in Dulbecco's Modified Eagle Medium (DMEM, 10\% FBS), and GJIC was evaluated using Calcein-AM loading as previously described [14]. Cells $(\mathrm{N}=30)$ were photo-bleached to $20 \%$ of original staining intensity using a $488 \mathrm{~nm}$ argon laser ( $80 \%$ power). Images were captured every 3 seconds until maximum recovery was reached (15 mins) using a LSM 800 Zeiss confocal microscopic using a 40x lens. Ten cytosolic areas $\left(2 \mathrm{~mm}^{2}\right)$ in bleached cell and unbleached non-adjacent cells were selected and mean fluorescence intensity was measured over the time course of the experiment using ImageJ Time Series Analyzer V3.

\section{Human Melanoma Biopsies}

Human melanoma samples and normal controls were provided by the Ontario Tumor Bank, which is funded by the Ontario Institute of Cancer Research (OICR). All specimens were verified by a pathologist at the OICR before use. Paraffin embedded sections (5 $\mu \mathrm{m})$ of primary cutaneous tumor $(\mathrm{N}=14)$, nodal melanoma metastases $(\mathrm{N}=15)$, and melanoma metastases from distant organ sites $(\mathrm{N}=7)$, in addition to normal controls $(\mathrm{N}=7)$ were selected. All work with human specimens was approved by The Human Science Research Ethics Board at the University of Western Ontario. Paraffin embedded sections were deparaffinized, subjected to antigen retrieval, and probed with rabbit anti-Cx43 (1:300; Sigma-Aldrich, C6219) or rabbit anti-MITF (1:200; 
Abcam, 20663) antibodies, overnight at $4^{\circ} \mathrm{C}$ prior to labeling with secondary antibodies as described above. To avoid the possible inclusion of adjacent normal tissue in analysis, 5-10 images of the tumor core and its surrounding radius of all samples was imaged using the 40x objective of the Zeiss LSM 800 confocal microscope. Images were then classified relatively as possessing no, low or high intensity of Cx43, and plaques were identified as punctate structures $(0.2-1 \mu \mathrm{m}$ in size) at the interface of two adjacent cells. Tissues were imaged blinded to the investigator to mitigate bias.

\section{Results and Discussion}

While it is abundantly clear that $\mathrm{C} \times 43$ is expressed in keratinocytes [4, 15], it's expression and role in melanocytes is far less defined. This problem is primarily due to the small population of melanocytes in the epidermis [16] and the difficulty in obtaining sufficiently high resolution immunolabeled images in situ to distinguish which skin cell types are truly expressing Cx43. To better elucidate whether $\mathrm{C} \times 43$ is expressed in melanocytes and the possible role of GJIC, we examined cultured mouse melanocytes for connexin expression and/or intercellular coupling. Primary mouse melanocytes were successfully isolated as evidence by positive expression for the melanogenesis markers, L-DOPA and TRP1 (Fig. 1A, B). Furthermore, the percent yield of L-DOPA positive melanocytes (dark pigment) was calculated across all passages (P2-P4), and the cultures were identified as $>90 \%$ melanocyte monocultures (Fig. 1A). While Cx43 positive plaques were readily detectable at the cell surface of rat epidermal keratinocytes (REKs) (Fig. 1C) and primary mouse keratinocytes before and after differentiation in the presence of high calcium (Fig. 1D), primary mouse melanocytes showed no evidence of Cx43 expression. To assess for intercellular coupling that could be facilitated by the presence of any member of the connexin family, keratinocytes and primary (P2) melanocytes were incubated with calcein-AM dye and dye recovery after photobleaching was measured. Primary melanocytes did not show any evidence of calcein-AM dye recovery after photobleaching compared to Cx43-positive REKs (Fig. 1E, F). Collectively, this indicates that primary mouse melanocytes are Cx43-negative and have no detectable levels of functional connexins capable of dye transfer.

If Cx43 fulfills the role of a tumor promoter in melanomas, one would predict that it would be up-regulated during disease onset and progression. The fate of $\mathrm{C} \times 43$ in melanoma progression and in distant metastasis are unclear [6-8]. However, our finding that primary melanocytes lack detectable
Cx43 and GJIC leaves open the possibility that Cx43 expression and gap junction assembly may be upregulated during melanoma tumorigenesis. Thus, to better characterize the role of Cx43 in human melanoma, the tumor core and surrounding radius of primary melanomas, in addition to nodal and distant metastases, were evaluated by immunofluorescence and imaging for low or high Cx43 expression and for the assembly of gap junctions. Consistent with Cx43 being absent from melanocytes, primary melanomas also showed no evidence of Cx43 expression (90\% of images from all donors), and in the rare cases where Cx43 was evident, it did not localize to gap junction plaques (Fig. 2A). As a control, Cx43 was readily detected in gap junctions in keratinocyte-rich normal skin (Fig. 2A). In addition, the majority of melanomas that metastasized to the lymph nodes did not express Cx43 (75\%), and while more Cx43 localized to intracellular regions in tumors defined as expressing high (9\%) and low (14\%) levels, few to no gap junction plaques could be identified (2\%) (Fig. 2B). Interestingly, the majority of samples collected from melanoma metastases to distant organs expressed Cx43 within intracellular compartments at both high (37\%) and low (34\%) levels. However, consistent with other stages of the disease, very few gap junction plaques were identified (8\%) (Fig. 2C). Furthermore, distant sites of metastasis which were also included in the Cx43 expression analysis (lung, femur, pelvic wall, kidney), stained positive for MITF confirming the tumor core and its surrounding radius was of melanocytic origin (Fig. 2D).

Using a library of human melanomas, we found that Cx43 protein was minimally expressed in primary cutaneous tumors and melanoma metastases to nodal tissue, but was expressed at moderate to high levels in melanoma metastases to distant organ sites. However, Cx43 expression remained largely intracellular; suggesting that any possible causal role Cx43 played in tumor progression would have to be gap junction-independent. Many gap junction-independent roles for $\mathrm{Cx} 43$ have been proposed but most involve the $\mathrm{C} \times 43$ interactome, which exceeds over 30 proteins, some of which have been linked to cancer or cell proliferation pathways, including NOV/CCN3, SRC, and caveolin -1 [17-19]. The inability of tumor cells to form $\mathrm{Cx} 43$-based gap junctions may be linked to a tumor survival mechanism, particularly to prevent the passage of molecules such as glutathione that assist in detoxifying carcinogens [20]. In our other studies, forced expression of $\mathrm{C} \times 43$ in B16-BL6 mouse melanomas resulted in increased GJIC which reduced the tumorigenic properties of the cells in both culture and in chicken chorioallantoic membranes [21]. If 
these mouse melanoma studies did indeed translate to the clinic this might suggest that $\mathrm{C} \times 43$ could be tumor suppressive in melanomas if it was successfully assembled into functional gap junctions; a process that is clearly defective in the human melanomas investigated here. Interestingly, it has been reported that Cx26 is upregulated in metastatic mouse melanomas where it could serve to form intercellular channels amongst melanomas or between melanomas and endothelial cells during extravasation [22]. Thus, Cx26 may be serving a very different role in melanoma tumorigenesis from $\mathrm{C} \times 43$ but targeting either or both connexins in advanced melanoma disease may have therapeutic advantages.
A

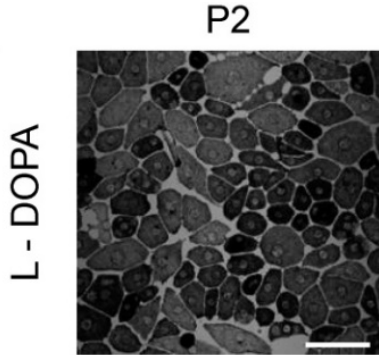

C

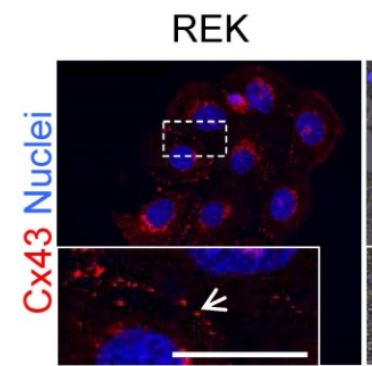

E

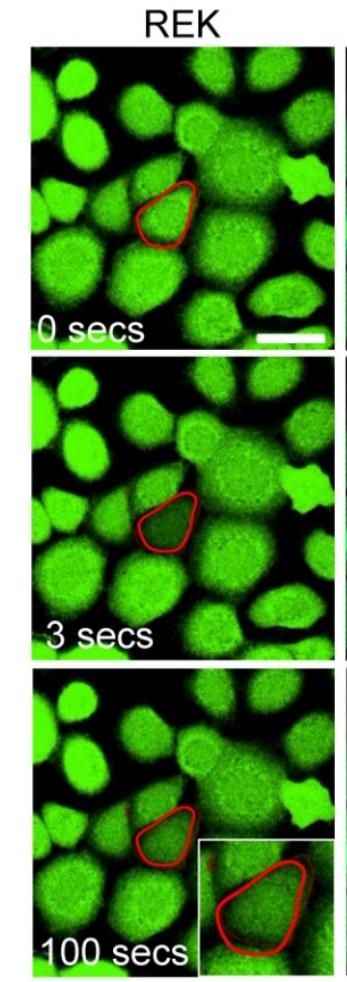

P3

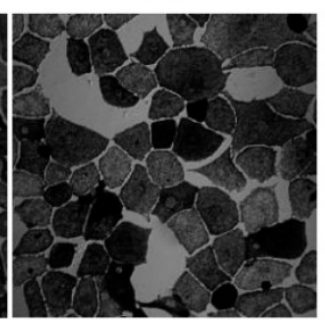

Melanocytes

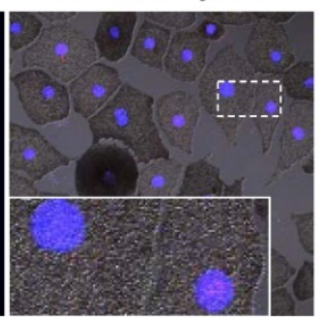

Melanocytes
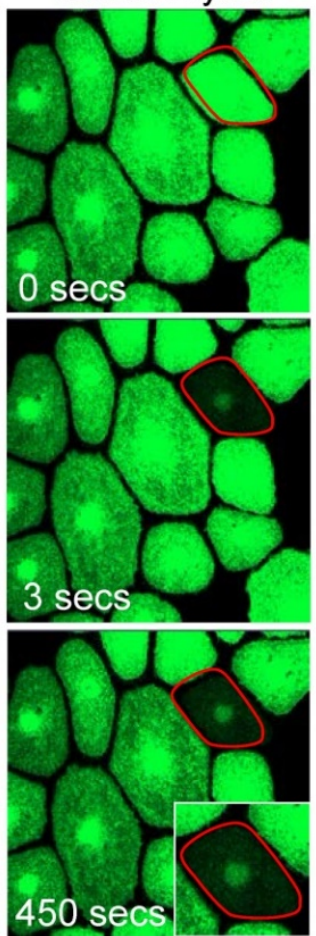
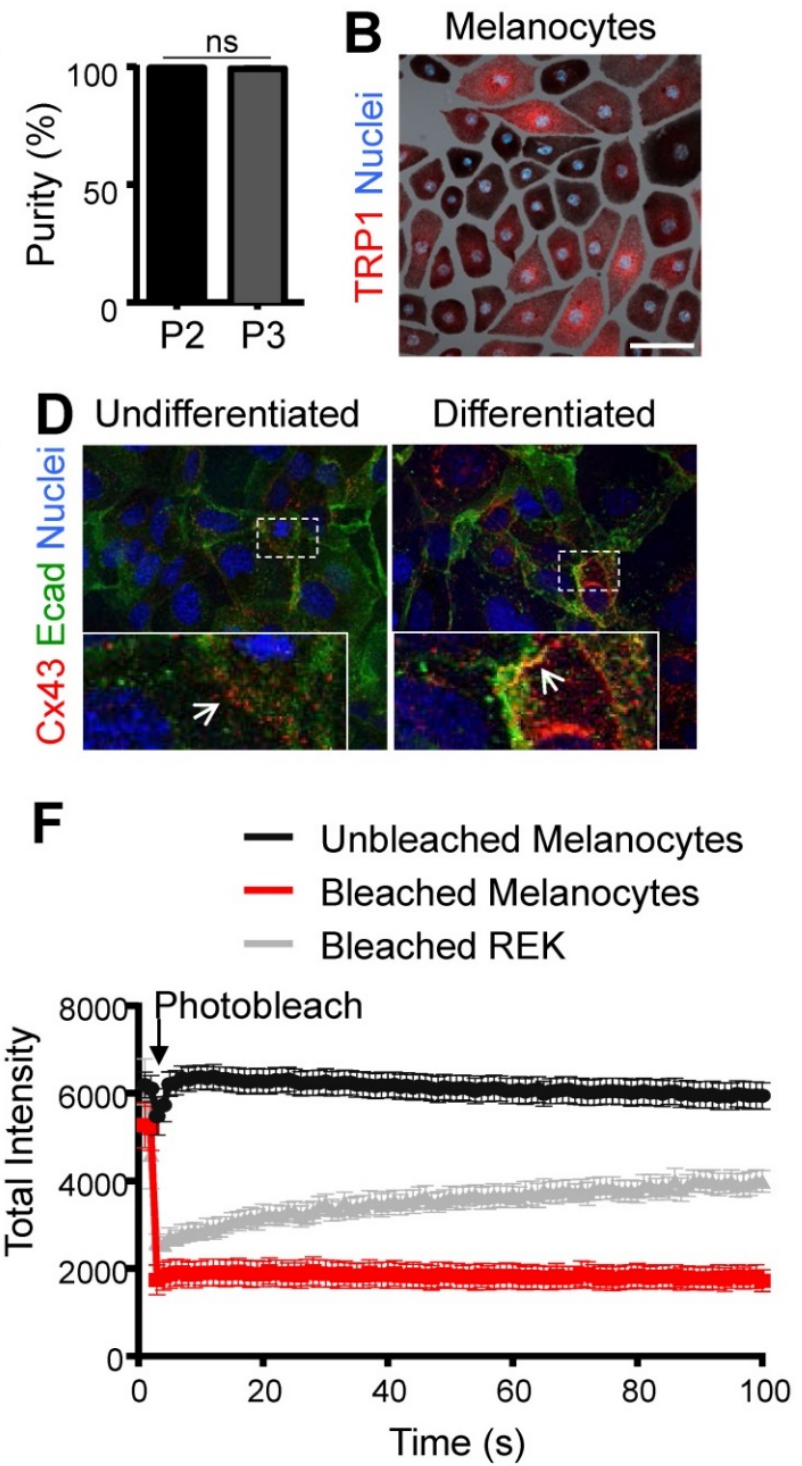

Figure 1: Primary mouse melanocytes show no evidence of Cx43 expression or GJIC Primary mouse melanocytes stained positive for L-DOPA (black pigment) (A) and were essentially pure across passage P2 and P3 (N=3,n=15), as revealed by staining for TRP1 (B; red). (C) Melanocytes exhibited no evidence of Cx43 expression, in comparison to Cx43-rich REKs, and (D) undifferentiated and differentiated primary mouse keratinocytes, which show evidence of Cx43 GJs (arrows) at the junctional membrane (E-cad= E-cadherin). (E) Outlined melanocytes loaded with calcein dye were photobleached and dye recovery was measured every 3 seconds for 450 seconds. In contrast to photobleached REKs $(N=3, n=30)$, photobleached melanocytes $(N=3, n=30)$ did not receive calcein dye indicating lack of GJIC. (F) Quantified data illustrates fluorescence recovery. Bars represent mean $+/$ - SEM. Scale bar $=20 \mu \mathrm{m}$. 

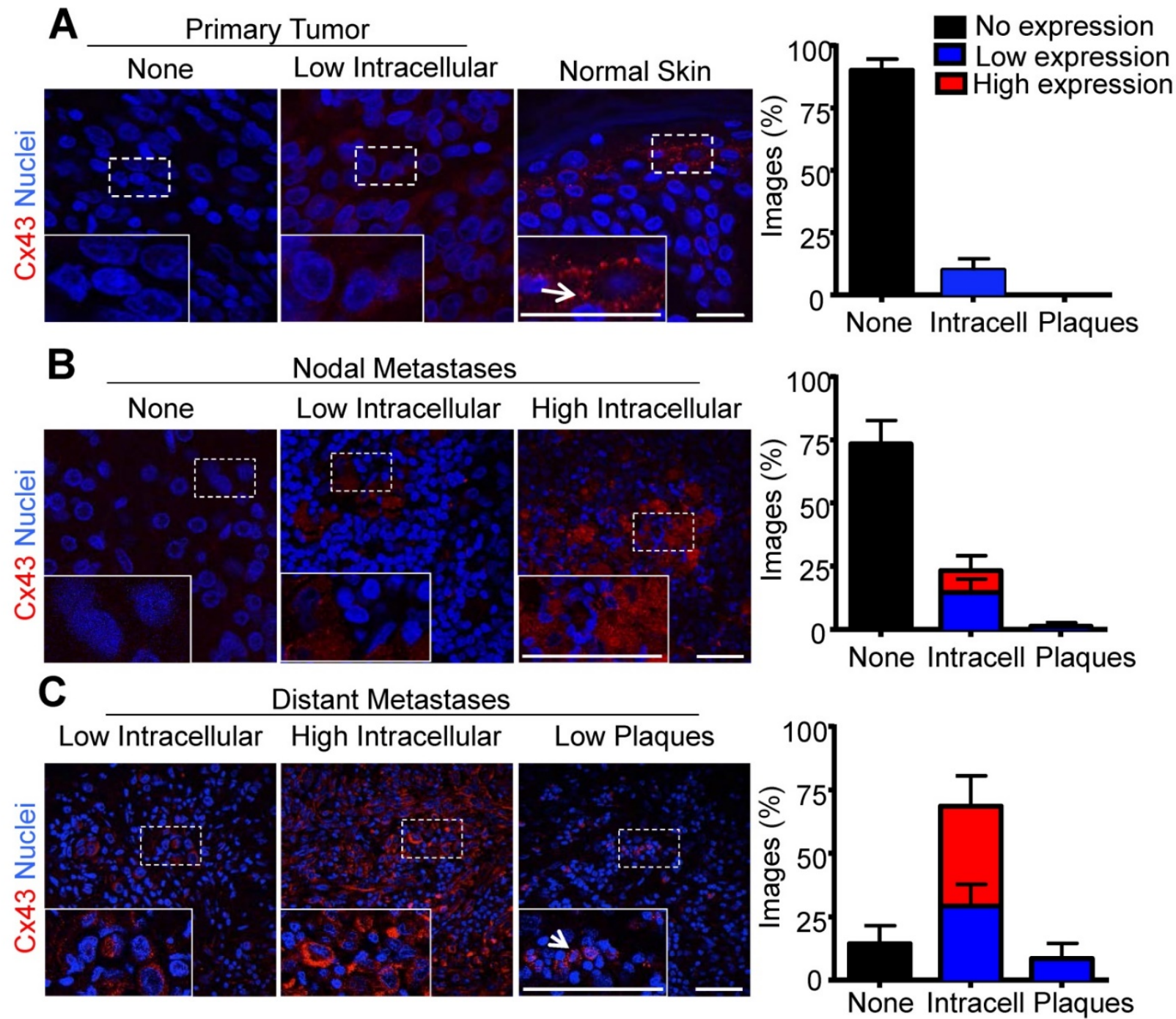

\section{D}

Distant Metastases
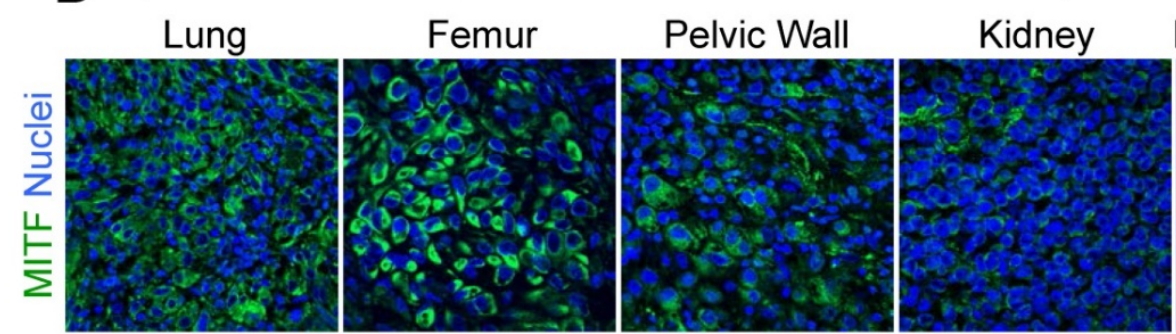

Normal Adjacent

Figure 2: Increased intracellular $\mathrm{Cx} 43$ in distant human melanoma metastases $(A)$ The majority of images analyzed from primary melanoma $(\mathrm{N}=14)$, and (B) nodal metastases $(N=15)$ did not show evidence of $C \times 43$ expression as opposed to normal skin where $\mathrm{Cx} 43$ gap junctions were abundant (A). In contrast, (C) the majority of images analyzed from melanoma metastases to distant organ sites $(\mathrm{N}=7)$ showed evidence of $\mathrm{Cx} 43$ expression, however, this expression was predominately intracellular and did not form gap junction plaque-like structures indicative of potentially functional gap junction channels. Arrows indicate punctate Cx43 gap junction structure at the cell-cell interface. (D) Regardless of the distant organ site of melanoma metastases, tumor cores from lung, femur, pelvic wall, and kidney stained positive for MITF, indicating the tumor was from a melanocytic cell lineage. Bars represent mean $+/$ - SEM. Scale bar $=20 \mu \mathrm{m}$.

Overall, we found that primary mouse melanocytes in monoculture did not express detectable Cx43 and are not GJIC dye-coupled with neighboring cells. This challenges the putative role of Cx43 as a potential regulator of melanocyte proliferation and tumor suppressor at early stages of melanocyte transformation. While we did not assess
Cx43 and GJIC in human melanocyte cultures, it was notable that nearly all primary human melanomas investigated $(\mathrm{N}=14)$ lacked $\mathrm{C} \times 43$ suggesting that even if there were very low levels of Cx43 in human melanocytes, they were quickly lost upon the onset of tumorigenesis. The general increase in intracellular Cx43 levels in melanomas that invaded lymph nodes 
followed by a further increase in intracellular Cx43 in melanomas that had distally metastasized correlates with the hypothesis that intracellular $\mathrm{C} \times 43$ is providing the tumor cells with a survival advantage but a causal relationship awaits further studies.

\section{Acknowledgements}

This work was supported by the Canadian Cancer Society (701459) to DWL and, in part, by the Canadian Institute of Health Research to DWL (123228). KCA was supported by The Cancer Research and Technology Transfer (CaRTT) Strategic Training Program. We would also like to thank Dr. Silvia Penuela for consulting on this project (The University of Western Ontario).

\section{Competing Interests}

The authors have declared that no competing interest exists.

\section{References}

1. Sohl G, Willecke K. An update on connexin genes and their nomenclature in mouse and man. Cell Commun Adhes. 2003; 10: 173-80.

2. Goodenough DA, Goliger JA, Paul DL. Connexins, connexons, and intercellular communication. Annu Rev Biochem. 1996; 65: 475-502.

3. Laird DW. Life cycle of connexins in health and disease. Biochem J. 2006; 394: $527-43$.

4. Churko JM, Laird DW. Gap junction remodeling in skin repair following wounding and disease. Physiology. 2013; 28: 190-8.

5. Hsu M, Andl T, Li G, Meinkoth JL, Herlyn M. Cadherin repertoire determines partner-specific gap junctional communication during melanoma progression. J Cell Sci. 2000; 113: 1535-42.

6. Rezze GG, Fregnani JH, Duprat J, Landman G. Cell adhesion and communication proteins are differentially expressed in melanoma progression model. Hum Pathol. 2011; 42: 409-18.

7. Sargen MR, Gormley RH, Pasha TL, Yum S, Acs G, Xu X, et al. Melanocytic Tumors Express Connexin 43 but not 26: Immunohistochemical Analysis With Potential Significance in Melanocytic Oncogenesis. Am J Dermatopathol. 2013; 35: 813-7.

8. Haass NK, Wladykowski E, Kief S, Moll I, Brandner JM. Differential induction of connexins 26 and 30 in skin tumors and their adjacent epidermis. J Histochem Cytochem. 2006; 54: 171-82.

9. Churko JM, Kelly JJ, Macdonald A, Lee J, Sampson J, Bai D, et al. The G60S Cx43 mutant enhances keratinocyte proliferation and differentiation. Experimental dermatology. 2012; 21: 612-8.

10. Kumar R, Parsad D, Kanwar A, Kaul D. Development of melanocye-keratinocyte co-culture model for controls and vitiligo to assess regulators of pigmentation and melanocytes. Indian J Dermatol Venereol Leprol. 2012; 78: 599-604

11. Wang D, Xu X, Ma H, Yue X, Li C, Zhu W. Optimization of the method for the culture of melanocyte precursors from hair follicles and their activation by 1,25-dihydroxyvitamin D3. Exp Ther Med. 2013; 6: 967-72.

12. Stewart MK, Gong XQ, Barr KJ, Bai D, Fishman GI, Laird DW. The severity of mammary gland developmental defects is linked to the overall functional status of Cx43 as revealed by genetically modified mice. Biochem J. 2013; 449: 401-13.

13. Baden HP, Kubilus J. The growth and differentiation of cultured newborn rat keratinocytes. J Invest Dermatol 1983; 80: 124-30.

14. Goldberg GS, Bechberger JF, Naus CC. A pre-loading method of evaluating gap junctional communication by fluorescent dye transfer. Biotechniques. 1995; $18:$ 490-7.

15. Langlois S, Maher AC, Manias JL, Shao Q, Kidder GM, Laird DW. Connexin levels regulate keratinocyte differentiation in the epidermis. J Biol Chem. 2007; 282: $30171-80$

16. Lin JY, Fisher DE. Melanocyte biology and skin pigmentation. Nature. 2007; 445: 843-50.

17. Laird DW. The gap junction proteome and its relationship to disease. Trends in cell biology. 2010; 20: 92-101.

18. Aasen T, Mesnil M, Naus CC, Lampe PD, Laird DW. Gap junctions and cancer: communicating for 50 years. Nat Rev Cancer. 2016; 16: 775-788.

19. Naus CC, Laird DW. Implications and challenges of connexin connections to cancer. Nature reviews Cancer. 2010; 10: 435-41.
20. Balendiran GK, Dabur R, Fraser D. The role of glutathione in cancer. Cell Biochem Funct. 2004; 22: 343-52.

21. Ableser MJ, Penuela S, Lee J, Shao Q, Laird DW. Connexin43 Reduces Melanoma Growth within a Keratinocyte Microenvironment and during Tumorigenesis in Vivo. J Biol Chem. 2014; 289: 1592-603.

22. Ito A, Katoh F, Kataoka TR, Okada M, Tsubota N, Asada H, et al. A role for heterologous gap junctions between melanoma and endothelial cells in metastasis. J Clin Invest. 2000; 105: 1189-97. 